\title{
Buenas prácticas de los centros de rehabilitación laboral (CRL) para personas con enfermedad mental grave de la Comunidad de Madrid a tener en cuenta para el diseño de un nuevo modelo de gestión de los servicios de empleo
}

Segundo Valmorisco*

Recibido: 05/06/2016 / Aceptado: 15/06/2016

Resumen. El presente artículo tiene como objetivo principal identificar las variables que explican por qué los Centros de Rehabilitación Laboral (CRL) para personas con enfermedad mental grave y duradera de la Comunidad de Madrid consiguen tasas de inserción sociolaboral cercanas al 50\%, siendo un colectivo que presenta unas tasas de desempleo superiores al $80 \%$.

La metodología empleada en la investigación del recurso (documental, cualitativa y cuantitativa) demuestra que estos resultados son posibles gracias a la conjunción de varios elementos: financiación sostenible, método de trabajo, fuerte liderazgo político, trabajo con el tejido empresarial (presentando a las empresas, perfiles profesionales basados en un certero análisis de las competencias), trabajo en red, plazo de intervención marcado por la persona y la composición de los equipos multiprofesionales, entre otros factores.

Palabras clave: Inserción sociolaboral, equipo multidisciplinar, itinerario individualizado, rehabilitación laboral, trabajo en red.

\section{Good practices of vocational rehabilitation centers (CRL) for people with} severe mental illness of the Community of Madrid to take into account for the design of a new management model for employment services

\begin{abstract}
This article aims to identify the variables that explain why the Vocational Rehabilitation Centres (CRL) for people with severe and enduring mental illness of the Madrid get labor insertion rates close to $50 \%$, with a group that presents rates unemployment above $80 \%$. The methodology used in the investigation of the resource (documentary, qualitative and quantitative ) shows that these results are possible thanks to the combination of several elements: sustainable financing, method of work and strong political leadership and work with the business community. This work is able to present the professional profiles companies based on a sound analysis of the skills and talents of its people served. More than half of employment intermediated by this resource belongs to standard companies. Other factors that explain these results are: networking, the term care is marked by the person or the composition of the multidisciplinary teams.
\end{abstract}

Keywords: Labor integration, multidisciplinary team, individualized itinerary, vocational rehabilitation, networking.

* Universidad Complutense de Madrid

svalmori@ucm.es 
Sumario. $1^{\circ}$. Introducción. $2^{\circ}$. Los centros de rehabilitación laboral (CRL): Características de población atendida y protocolo de actuación. $3^{\circ}$. Factores de éxito de los CRL. $4^{\circ}$. Enseñanzas y recomendaciones de este recurso para el diseño de modelos de gestión en servicios de empleo. $5^{\circ}$. Orientación metodología. $6^{\circ}$. Bibliografía.

Cómo citar: Valmorisco, S., «Buenas prácticas de los centros de rehabilitación laboral (CRL) para personas con enfermedad mental grave de la Comunidad de Madrid a tener en cuenta para el diseño de un nuevo modelo de gestión de los servicios de empleo», en Cuadernos de Gobierno y Administración Pública 3-1, 00-00.

\section{Introducción}

En nuestra sociedad actual (de la hipermodernidad según Guilles Lipovetsky, marcada por la obsesión por la inmediatez, el hiperconsumo de bienes y servicios y la hiperestimulación), existe una justificada urgencia, tanto por parte de la oferta (para dar respuesta a las necesidades de incorporación de personal a las plantillas de las empresas) como por parte de la demanda (con inaceptables tasas de desempleo en España en los últimos años) por ajustar ambas solicitudes de la forma más rápida posible. La burocracia (tal y como plantea en sus escritos Max Weber) se convierte en un sistema de organización, pero sobre todo de dominación, que causa el mayor obstáculo en este proceso. Las disfunciones de la propia burocracia, identificadas por Robert K. Merton (excesivo apego a reglas y reglamentos, incapacidad adiestrada, despersonalización de las relaciones humanas) parecen ser cómplices de un mercado incapaz de dar respuesta a la gran demanda actual de puestos de trabajo y convierten al ciudadano en un ser indefenso frente a la Administración Pública. En la actualidad, muchos servicios de empleo públicos, continúan en un claro retroceso respecto a políticas de empleo exitosas desarrolladas en España a finales de los años 90, cuando llegaron una considerable cantidad de recursos económicos provenientes del Fondo Social Europeo (FSE) para hacer frente a este tipo de políticas. En esos años, se daba una más pronta y certera respuesta a las demandas de personal de las empresas que la que damos hoy en día. Este retroceso, se explica sobre todo, por la falta de una respuesta técnica, lo que hace que se elaboren protocolos consistentes en laberintos interminables de solicitudes, permisos y autorizaciones que, una vez más, maltratan y desatienden a la oferta de las empresas, lo que trae consigo falta de respuesta hacia una demanda de empleo cada vez más empobrecida, con el añadido que esas situaciones de pobreza actualmente trascienden a la propia demanda, afectando incluso a la población ocupada, los denominados "working poor".

En tiempos de crisis económica y de francas dificultades para crear empleo estable y de calidad, como vivimos en estos momentos, se hace más necesario que nunca identificar recursos que puedan dar respuesta a esta urgente necesidad.

El desempleo es un síntoma del anormal comportamiento de nuestro mercado de trabajo. No deberíamos acostumbrarnos a tasas de desempleo superiores al $20 \%$ que son causadas por diversos factores no corregidos a lo largo del tiempo en España. En el caso de las personas jóvenes y mayores de 45 años, estas tasas suponen varias veces la media de la UE.

Revisando los datos de la Encuesta de Población Activa (EPA) del Instituto Nacional de Estadística (INE), difícilmente podíamos imaginar en el segundo tri- 
mestre de 2007 que siete años después, nuestra tasa de desempleo afectaría a casi 6 millones de personas, llegando a una tasa cercana al $26 \%$ de la población activa (primer trimestre de 2014). En aquellos momentos, nos situábamos en una cifra más de tres veces inferior a la actual, con un histórico 7,93\% de paro que afectaba tan sólo a 1,77 millones de personas, por detrás de países como Francia y Alemania y tan sólo a un punto porcentual de la media de la UE. En aquel momento trabajaban en España 20,58 millones de personas, hoy en día a duras penas alcanzamos los 17 millones.

¿Qué hemos hecho mal en estos años para que nuestro mercado de trabajo arroje el peor comportamiento, junto a Grecia, de toda la Unión Europea?

La actualidad nos exige concentrar nuestros esfuerzos en identificar posibles soluciones a la situación actual, a partir de la investigación de recursos de empleo que obtengan buenos resultados de inserción sociolaboral, como es el caso de los Centros de Rehabilitación Laboral (CRL) para personas con enfermedad mental grave de la Comunidad de Madrid. Según datos de la Red pública de atención social a personas con enfermedad mental grave, correspondientes a 2014, dicho recurso consigue una tasa de inserción laboral del 50\%, frente al 1,7\% que en 2015 consiguió intermediar el Servicio Público de Empleo Estatal (SEPE) según datos de la Encuesta de Población Activa (EPA) correspondientes a 2015. Esta cifra de inserción nos ofrece un resultado más que positivo, sobre todo si nos referimos a colectivos en grave riesgo de exclusión social como es el caso de las personas con enfermedad mental, quienes soportan una tasa de desempleo de partida es del $80 \%$, por lo que se hace más necesario analizar en profundidad las variables que hacen posible este "milagro".

Como hemos señalado anteriormente, nuestro país ha recibido importantes ayudas provenientes del FSE para promover la creación de empleo. A partir de mediados de los años 90 , se inyectó gran cantidad de recursos económicos en favor de la cualificación de la demanda (sobre todo en las fases de orientación y formación de sus itinerarios de inserción sociolaboral). Los orígenes de los servicios de empleo para personas con enfermedad mental grave en la Comunidad de Madrid (actuales $\mathrm{CRL}$ ) provienen de este momento, si bien el diseño definitivo es posterior. De manera generalizada, podemos decir que los organismos que gestionaron estos fondos, no supieron dar respuesta a dos problemas que aún siguen sin solucionarse:

1) No consiguieron extraer suficientes soluciones metodológicas.

2) No consiguieron consolidar su gestión con el tejido empresarial.

Habiendo empeorado nuestro actual contexto laboral y siendo más necesario que nunca crear puestos de trabajo de calidad, debemos preguntarnos cuales deberían ser las premisas para que los servicios de empleo cumplan su cometido.

Para abordar esta doble realidad: personas con enfermedad mental grave y recursos o estructuras, como son los Centros de Rehabilitación Laboral donde se desarrollan los itinerarios de inserción sociolaboral, vamos a comenzar por entender cada una de estas dimensiones. Para ello, vamos a esbozar unas ideas iniciales, para después cuantificar el número de personas a quienes afecta esta enfermedad y sus características básicas, así como el protocolo de actuación que llevan a cabo estos centros. 


\section{Los centros de rehabilitación laboral (CRL): Características de la pobla- ción atendida y protocolo de actuación}

Como hemos indicado, el primer CRL es fundado doce años antes de que esta crisis económica y financiera comenzase a afectarnos de manera directa. Nos remontamos a 1996, año en que algunos técnicos de la Comunidad de Madrid se plantean alternativas al internamiento psiquiátrico de miles de personas, ofreciendo herramientas de inserción socio-laboral que complementasen los tratamientos de salud mental y los psicofármacos.

La Red pública de atención a personas con enfermedad mental grave y duradera de la Comunidad de Madrid, ofrece en la actualidad 6.064 plazas en diferentes recursos para el colectivo (centros de rehabilitación psicosocial, centros de día de soporte social, centros de rehabilitación laboral, residencias, pisos supervisados, etc.). En concreto, los CRL atienden a un total de 1.351 personas, de las cuales el $50 \%$ encuentran empleo (676 personas con enfermedad mental grave y duradera).

Los CRL, constituyen el recurso social especifico, (dependientes del Programa de Atención social a Personas con enfermedad mental crónica), cuya misión fundamental es favorecer la rehabilitación vocacional-laboral de las personas con trastornos psiquiátricos crónicos que se encuentran viviendo en la comunidad, de modo que propicie su integración laboral normalizada fundamentalmente en la empresa ordinaria o también en fórmulas de empleo protegido (Centros especiales de empleo) o sistemas de auto-empleo. Los CRL no tienen carácter de centro de trabajo, por lo que no pueden participar en operaciones regulares de mercado ni incluir relaciones laborales remuneradas con sus usuarios, como si lo hacen otros recursos tales como los centros especiales de empleo o las empresas de inserción, donde si es posible establecer relaciones de trabajo con las personas que pertenecen a dichos centros, asi como entre estas entidades "empresariales» y otras empresas para la prestación de bienes o servicios.

En la actualidad existen en la Comunidad de Madrid 21 CRL. Todos ellos compuestos por un equipo multiprofesional formado por: Director (psicólogo), 1 psicólogo, 1 Terapeuta Ocupacional, 1 Técnico de Apoyo a la Integración Laboral, 1 Preparador Laboral, 3 Maestros de Taller, 1 Auxiliar Administrativo, 1/2 auxiliar de limpieza.

\section{1. ¿A qué tipo de población atiende este recurso?}

El colectivo de personas con enfermedad mental grave presenta diferencias en cuanto a las condiciones de partida de cada una de las personas atendidas, dichas condiciones ejercen una influencia directa en sus resultados de inserción: tipo de enfermedad mental, ingresos económicos de los hogares, nivel de funcionamiento psicosocial, estado civil, experiencia laboral previa, nivel académico, etc.

En concreto, el perfil-tipo de los usuarios/as que acuden a los CRL es el siguiente: todas las personas presentan enfermedad mental grave y duradera, siendo la esquizofrenia, otros trastornos psicóticos y los trastornos de personalidad los diagnósticos clínicos más frecuentes, en más de $70 \%$ de los casos tienen ingresos económicos propios procedentes de: su propio trabajo, pensión no contributiva o prestación por hijo a cargo. Casi el $90 \%$ son personas solteras con un nivel académico 
de Enseñanza primaria. EGB ( $2^{\text {a }}$ Etapa: $8^{\circ}$ ) o Bachiller Superior, BUP, COU, Bachillerato y con alguna experiencia laboral previa.

Según el Centro de Investigación Biomédica en red de Salud Mental (Ministerio de Ciencia e Innovación del Gobierno de España e Instituto de Salud Carlos III), en España se considera que el 9\% de la población padece en la actualidad algún tipo de trastorno relacionado con la salud mental.

Según el Estudio ESEMeD-España 2006 (dicho estudio es parte de un proyecto europeo sobre epidemiología de los trastornos mentales, enmarcado dentro de una iniciativa de la World Mental Healt Surveys de la Organización Mundial de la Salud, llamada Encuestas de Salud Mental en el mundo) el 19,5\% de las personas presentaron un trastorno mental en algún momento de su vida (prevalencia-vida) y un $8,4 \%$ en los últimos 12 meses (prevalencia-año).

El trastorno mental más frecuente es el episodio depresivo mayor, que tiene un $3,9 \%$ de prevalencia-año y un $10,5 \%$ de prevalencia-vida. Después del episodio depresivo mayor, los trastornos con mayor prevalencia-vida son la fobia específica, el trastorno por abuso de alcohol y la distimia. Los factores asociados a padecer un trastorno mental según perfiles son el sexo femenino, estar separado, divorciado o viudo, estar desempleado, de baja laboral o con una incapacidad.

Según los últimos datos se estima que las enfermedades mentales consumen hasta el $20 \%$ del gasto sanitario en los países desarrollados, con una previsión al alza en los próximos años (XVII Simposio de la Fundación Lilly, 2010).

Es muy importante tener en cuenta que la disponibilidad de las personas hacia el empleo, así como otras variables añadidas: autonomía, independencia, emancipación familiar, etc. es muy diferente según sea su:

- Género, las mujeres están sujetas a una doble exclusión en el caso de la discapacidad. «En términos relativos, el 17,3\% de las mujeres con discapacidad se encontraba en situación de pobreza extrema, tasa que triplicaba la de la población española $(6,4 \%)$ en 2008 y era sensiblemente superior a la de los varones con discapacidad (11\%). El riesgo de pobreza no presenta diferencias significativas por género entre personas con discapacidad, aunque es prácticamente el doble para este grupo poblacional que para el resto de la población $(51 \%$ frente a 26\%)" (Huete, 2013: 29)

- Edad, la generación de los mayores de 45 años, quienes tenían 16 y más años en 1982, fecha de aprobación de la LISMI.

- Nivel académico, generalmente, como ocurre con el mercado de trabajo en general, a más nivel académico mayor tasa de empleo. Según la Encuesta de Discapacidad, Autonomía Personal y Situaciones de Dependencia (EDAD) elaborada por el INE, De los casi 3,9 millones de personas con discapacidad, 198.600 tienen estudios universitarios o equivalente finalizados, esto es, sólo un $5,16 \%$ de la población con discapacidad alcanza el nivel educativo superior. Si se analiza la población entre 6 y 64 años el porcentaje alcanza el $7,88 \%$, siendo sólo el $0,84 \%$ en la población entre 6 y 24 años. Según esta misma fuente, el $83,83 \%$ de la población femenina con deficiencia mental (suma del conjunto de personas con enfermedad mental más las personas con discapacidad intelectual) posee estudios inferiores a educación secundaria, siendo en el caso de los varones del 72,67\%. 
- Actitud y cultura hacia el empleo, variable en la que juega un papel fundamental la familia y el entorno.

- Tipo y grado de discapacidad, variable que puede limitar en mayor medida que el resto la capacidad de la persona para ocupar un puesto de trabajo con unas mínimas garantías.

\section{2. ¿Cuál es el protocolo de actuación de los CRL?}

El protocolo de actuación que sigue este recurso de empleo es el de la gestión de itinerarios de inserción socio-laboral. En la actualidad no existe un claro consenso en relación a las fases que contienen dicho itinerario, tanto desde el ámbito de los expertos como de algunas de las entidades sociales más especializadas (por ejemplo, Fundación Tomillo y Fundació Pere Tarrés).

En este sentido, podría decirse que todos los servicios de empleo, tanto públicos, como privados: ONG, Sindicatos, Patronal, Administración pública y Universidades, hacen uso de su propio itinerario, adaptando en cada caso, y en ocasiones sin un modelo teórico claro, las diferentes fases a cada persona, sin que podamos hablar de un protocolo cerrado ni consensuado para ello. Podemos considerar, que estos seudo-modelos adolecen en muchos casos de deficiencias metodológicas que posiblemente afecten a su resultado final.

Antes de seguir con las fases del itinerario, es importante añadir que el canal de entrada de las personas usuarias en este recurso, son los centros de salud mental de distrito, de quienes se recibe la primera derivación, lo que da cuenta de una relación multinivel desde el propio inicio de la atención.

Cuadro 1. Itinerario de inserción sociolaboral aplicado en los centros de rehabilitación laboral (CRL)

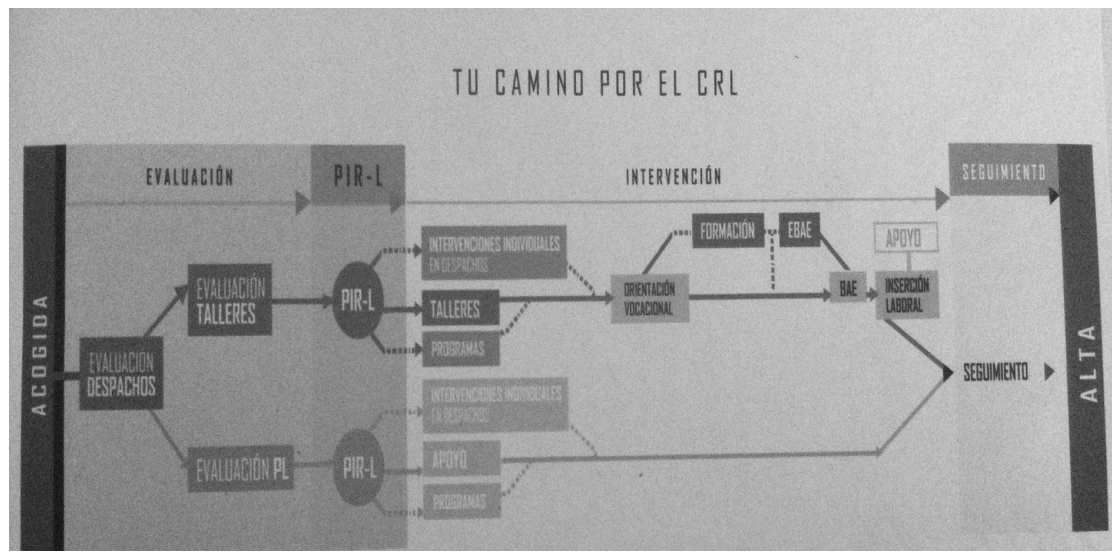

El modelo aplicado por los CRL presenta la novedad de incorporar la evaluación nada más terminar la fase de acogida, no así al terminar cada una de las diferentes fases del total del proceso (como ocurre con otros modelos, como el de la Fundación Tomillo). Esta fase de evaluación se realiza por cada uno de los profesionales 
que componen el equipo de trabajo (psicólogo, TAIL, preparador laboral, terapeuta ocupacional y maestros de taller), así como se evalúa el comportamiento de cada persona en los talleres pre-laborales durante su estancia previa de 45 días. Es necesario apuntar un par de cuestiones: a) anualmente se realiza una evaluación de los objetivos con la persona atendida y estos pueden cambiar en función de la misma y b) Este itinerario no es lineal, sino que ofrece en la práctica bastante flexibilidad, de modo que una persona puede pasar del PIR-L a la fase de formación o directamente al empleo si así lo aconsejan las circunstancias.

Si bien existen en el modelo dos puntos de evaluación del proceso (una evaluación que se hace manifiesta en los primeros 45 días de la persona en el recurso y otra al final del itinerario), consideramos que un exceso de evaluación no garantiza el éxito de la inserción laboral, como tampoco es crucial evaluar al final de un proceso tan largo, así como no contar con el feed-back de la información por parte de la persona.

Una vez terminado este primer proceso evaluador, posterior a la fase de acogida, se convoca una Junta de evaluación, en la que participan todos los profesionales del CRL que han analizado la inmersión de la persona en los diferentes talleres. Con toda esa información se establece y propone el Plan individualizado de recuperación laboral (PIRL). Este PIRL se remite a salud mental, organismo de quien se recibe la derivación de la persona.

En definitiva, las fases de forma resumida son: acogida, evaluación, PIRL, intervención, seguimiento y alta, siendo la segunda fase la que presenta diferencias con cualquier otro modelo estudiado.

Como podemos comprobar en la imagen anterior (Itinerario de los CRL) existen dos rutas bien diferenciadas: a) La ruta superior se lleva a cabo entre las personas que acuden al CRL y no tienen empleo y b) La ruta inferior se lleva a cabo entre las personas que acuden al recurso con un puesto de trabajo y precisan de herramientas para mantenerlo. Este segundo grupo tienen como profesional de referencia al preparador laboral, quien realiza tareas de Empleo con apoyo. Las personas atendidas que se encuentran en este itinerario no acuden a los talleres, pero se les hace un PIRL y se les ofrece apoyo, así como los programas propios de cada centro. El itinerario para este segunda ruta sigue con las fases de seguimiento (puesto que la persona ya tiene un empleo) y alta.

En la ruta superior, una vez terminado el periodo de 45 días que culmina con la elaboración compartida junto a la persona de su Plan Individualizado de Rehabilitación Laboral (PIR-L), plan en el que se marcan sus objetivos a conseguir, comienza la fase de intervención en la que la persona se incorpora de forma definitiva a los talleres y programas del centro, así como se continúan con las intervenciones individuales en despachos con cada uno de los profesionales. Esta fase de intervención abarca: trabajo en programas y talleres (así como intervenciones individuales en despachos), Orientación vocacional, formación, Entrenamiento en Búsqueda Activa de Empleo (EBAE), BAE, Inserción laboral y empleo con apoyo.

Otra característica importante de este modelo, es que la fase de orientación vocacional se encuentra ya en el propio proceso de intervención, y no antes, como así ocurre en la mayoría de modelos, de esta forma se asesora para "pulir" mejor la identificación de capacidades e intereses laborales de la persona. Esta fase es anterior a la fase de formación y de búsqueda activa de empleo (BAE) y a la fase de intermediación laboral, es decir, la persona atendida ya participa de los diferentes talleres y aprende haciendo. En esta fase de orientación vocacional, el profesional de referencia es el terapeuta ocupacional. 
El siguiente proceso en la fase de intervención, es el de identificar las necesidades de formación de la persona, formación que generalmente se realiza fuera del propio centro. A partir de este proceso la persona se centra en el empleo, comenzando con el Entrenamiento en Búsqueda Activa de Empleo y con la propia BAE. Posteriormente le sigue la fase de Inserción laboral con empleo con apoyo.

En ambos casos (ruta superior y ruta inferior) el itinerario termina con la fase de seguimiento de la persona insertada y con la fase de alta.

Debemos considerar la importancia de los itinerarios de creación de empleo para cualquier persona que esté buscando empleo. Dependiendo de los perfiles profesionales, es posible obviar alguna de sus fases, sin embargo, dadas las especiales características de las personas con enfermedad mental, no es conveniente modificar el esquema-modelo del mismo (y mucho menos eliminar alguna de sus fases), en este sentido la fase de orientación laboral-vocacional no debe realizarse únicamente sobre la base de lo que el sujeto nos trasmite como preferencias de ocupación, hemos de dedicar el tiempo necesario, y más con quien padece una enfermedad mental, para ayudarle a organizar y reorientar sus preferencias desde un punto de vista objetivo, aparte de sus preferencias y experiencia laboral previa.

Una vez conocidas las características de la población atendida por este recurso y las diferentes fases del itinerario de inserción sociolaboral o protocolo seguido por dicho recurso, vamos a analizar cuáles son los factores de éxito que posibilitan los resultados señalados.

\section{Factores de éxito de los CRL}

Las altas tasas de inserción sociolaboral de los CRL de la Comunidad de Madrid son producto de la conjunción de varios factores: la ubicación del recurso en el ámbito de los servicios sociales (si bien debería establecerse una mayor conexión con el área de empleo); el liderazgo de los técnicos de alto nivel y su coordinación con las direcciones generales y consejerías implicadas, la alta competencia, vocación y motivación de los equipos profesionales, así como la fórmula de financiación a través del concierto (con unos pliegos que especifican al detalle las condiciones técnicas).

El éxito de este modelo de gestión, se explica por todos estos factores y especialmente en el análisis comparativo de los diferentes recursos en las CC.AA por: a) la coordinación con salud mental en la derivación de casos, b) la gestión compartida con la persona de las diferentes fases del itinerario, c) la armonizada composición del equipo de trabajo (especialmente con la figura del Técnico de Apoyo a la Inserción Laboral), d) la apuesta de la Comunidad de Madrid al mantener el presupuesto, e) el liderazgo de los técnicos de alto nivel y su coordinación con las direcciones generales y consejerías implicadas, son algunos de los factores que explican el éxito del modelo.

\section{Factores que facilitan la inserción laboral:}

Desde el recurso de los CRL se ha comprobado que los factores de éxito para la inserción laboral de las personas usuarias son: 
i. Motivación de la persona.

ii. Apoyo familiar recibido.

iii. Experiencias laborales previas.

iv. Buen nivel de funcionamiento (habilidades y capacidades).

Todos estos aspectos son trabajados en los CRL, a excepción de las experiencias laborales previas. No obstante, se pulen las competencias adquiridas en el pasado y se adaptan las mismas, así como se adquieren otras nuevas a través de los diferentes programas en los que la persona participa.

Tal y como hemos podido comprobar en la investigación cualitativa realizada (en la que, como indicaremos más adelante, se han realizado 6 grupos de discusión y 6 entrevistas en profundidad), la información contenida en este artículo es producto de mi tesis doctoral, "Políticas públicas de rehabilitación laboral para personas con enfermedad mental grave- Los Centros de Rehabilitación Laboral (CRL) en la Comunidad de Madrid (2008-2012)", leída en marzo de 2015. Algunos de estos mismos agentes podrían inhibir la inserción sociolaboral de las personas atendidas, a saber: no sólo la propia persona y la familia, sino también la sociedad y el propio equipo profesional. De modo que podríamos situar cuatro agentes claros en el proceso de rehabilitación laboral:

\begin{tabular}{c|c} 
PERSONA & FAMILIA \\
& \\
\hline $\begin{array}{c}\text { SOCIEDAD - POLÍTICAS } \\
\text { (Micro y Macro) }\end{array}$ & EQUIPOS PROFESIONALES \\
\end{tabular}

Por otro lado, como ya hemos indicado, otro factor de éxito tiene que ver con que la crisis no ha afectado a la estabilidad del recurso, como lo ha hecho en otras CC.AA, en la medida en que este modelo ha estado protegido tanto en efectivos como en dotación presupuestaria por la propia Comunidad de Madrid. Bien es cierto que la Red de Atención Social a personas con enfermedad mental ha sufrido un ajuste presupuestario, así en el periodo 2012-2013 el presupuesto se redujo en casi un 7\%, pasando de los 64,7 millones en 2012 a los 60,3 millones en 2013. No obstante, hemos de decir que a pesar de este ajuste y de una coyuntura desfavorable, el número de plazas se ha incrementado en el periodo estudiado, e incluso en la actualidad sigue esta tendencia al aumentar en nuevas plazas en 2014 (hasta llegar a las 1.351 existentes en la actualidad), así como el propio presupuesto que se incrementa en este último año en más de un $4 \%$. 
Tabla $1 . \mathrm{N}^{\mathrm{o}}$ plazas y presupuesto red de atención social a PEM GRAVE comunidad de Madrid (2008-2014)

\begin{tabular}{|c|c|c|c|c|c|c|c|c|}
\hline & $\mathbf{2 0 0 8}$ & $\mathbf{2 0 0 9}$ & $\mathbf{2 0 1 0}$ & $\mathbf{2 0 1 1}$ & $\mathbf{2 0 1 2}$ & $\mathbf{2 0 1 3}$ & $\mathbf{2 0 1 4}$ & Incremento \% \\
\hline Número total plazas de la Red & 5.260 & 5.350 & 5.518 & 5.578 & 5.679 & 5.754 & 6.064 & $15,28 \%$ \\
\hline Número total plazas CRL & 977 & 1.104 & 1.137 & 1.260 & 1.313 & 1.330 & 1.351 & $38,28 \%$ \\
\hline PRESUPUESTO TOTAL (Mill $€$ ) & 56 & 59 & 60,6 & 63,6 & 64,7 & 60,3 & 62,9 & $12,32 \%$ \\
\hline
\end{tabular}

Fuente: Red de Atención social a Personas con enfermedad mental grave. Comunidad de Madrid.

A los factores indicados (financiación sostenible, método de trabajo y fuerte liderazgo político) hay que añadir uno no menos importante: el trabajo con el tejido empresarial. Dicho trabajo consiste en poder presentar a las empresas perfiles profesionales basados en un certero análisis de las competencias y el talento de sus personas atendidas. Más de la mitad del empleo intermediado por este recurso pertenece a empresas normalizadas. Otro factor que debemos añadir es el trabajo en red de todos los CRL quienes comparten ofertas de empleo, protocolos de actuación, etc estableciendo reuniones mensuales de coordinación.

La perspectiva comparada con otros recursos de empleo para personas con enfermedad mental grave en países anglosajones, nos permite establecer una perspectiva comparada del recurso. El modelo CRL está basado en el modelo «primero entrena y después coloca», por el contrario el modelo IPS (Individual Placement and Support) se basa en el "primero coloca y después entrena" (desarrollado en EEUU y Reino Unido). La comparativa entre ambos nos permite aseverar que el modelo CRL presenta suficientes evidencias empíricas (con un volumen de casos 3,3 veces superior respecto al modelo IPS) como para poder probar su eficacia dada la tasa de inserción laboral conseguida (50\%), tasa tan sólo 5 puntos porcentuales inferior a la conseguida por el modelo IPS (55\%) y todo ello a pesar de la existencia de unos factores externos claramente desfavorables: mayores tasas de desempleo existentes en España, inferior renta per cápita disponible de las familias y mayor proteccionismo de las propias familias y mayor riesgo de las "pensiones trampa" en un sistema de corte conservador.

Una vez expuestos los principales factores de éxito de este recurso, en el siguiente apartado vamos a enumerar algunas enseñanzas y recomendaciones que el modelo CRL para personas con enfermedad mental grave de la Comunidad de Madrid, puede mostrar a otros modelos de gestión de servicios de empleo, ya sean dedicados a colectivos en riesgo de exclusión social o no.

\section{Enseñanzas y recomendaciones de este recurso para el diseño de modelos de gestión en servicios de empleo}

- Una primera enseñanza extraída de este análisis comparativo del recurso en las diferentes Comunidades Autónomas, es la falta de liderazgo por parte de la Administración del Estado para coordinar unas competencias que, precisa- 
mente porque pertenecen a las CC.AA, reproducen un auténtico galimatías, al establecerse 17 modelos diferentes de atención (uno diferente para cada Comunidad Autónoma, sin establecerse unos criterios metodológicos que identifiquen y justifiquen su estructura). Este hecho puede hacerse extensible a nuestras políticas de empleo. Bien este cierto, que existen multitud de variables que interaccionan entre sí y en ocasiones impiden su buen desarrollo, y más en un país tan diverso como es España: distintas densidades de población, diferentes formas de concebir el modelo, atomización poblacional, etc. todo ello contribuye a que cada CC.AA actúe de forma particular.

- Recomendamos la creación de una gran base de datos que abarque a las personas atendidas en los diferentes dispositivos de rehabilitación laboral (en todas las fases del proceso), de modo que se identifiquen en la misma las variables objeto de un amplio estudio cuantitativo. Consideramos que por los resultados alcanzados en inserción laboral (recordemos que su tasa de inserción es probablemente la más alta de España) se debería liderar desde los CRL un gran estudio a nivel nacional que aporte evidencias empíricas e identifique todos los factores de éxito en las diferentes CC.AA, y recomiende en cada caso el itinerario y los modelos de rehabilitación laboral más convenientes. Una gran base de datos a nivel nacional de las personas demandantes de empleo conseguiría romper las fronteras regionales en la gestión de las ofertas. Esa base debería casar oferta y demanda con un análisis específico de los perfiles solicitados.

- Se recomienda un análisis en profundidad que identifique las competencias que abarcan cada puesto de trabajo, para orientar la I+D de los CRL en la consecución de esas competencias mediante adaptaciones. Esta recomendación podría hacerse extensible a todos los colectivos, debiendo convertirse en la hoja de ruta de los itinerarios de inserción sociolaboral de las personas atendidas en los servicios de empleo.

- Se recomienda en el nivel nacional (no siendo el caso de los CRL de la Comunidad de Madrid) una financiación estable para evitar que los equipos profesionales y los servicios ofrecidos fluctúen y no puedan garantizar la atención de las personas atendidas.

Obviamente y como acabamos de indicar, todo ello debe ser apoyado económicamente creando un presupuesto anual para tal fin, de esta forma se lograría equiparar y nivelar los diferentes servicios prestados por cada recurso. Pero todo esto no sería posible sin un amplio consenso y puesta en común de todas y cada una de las Administraciones regionales, un consenso al estilo del realizado en 1990 para la elaboración del Plan Concertado de Servicios Sociales.

En este sentido, se ha constatado que la Comunidad de Madrid ha logrado importantes consensos para implantar este recurso multinivel, el cual abarca diversas áreas, como son: empleo, formación, servicios sociales y salud, dentro de la Administración Regional, pero que también involucra a empresas y Asociaciones y Fundaciones, todas ellas persiguiendo un mismo fin, a sabiendas de los beneficios que produce, en este colectivo, la inserción en el mercado de trabajo. Todo ello da cuenta de una colaboración público-privada que ofrece, desde el modelo concertado, una solución para aquellas personas con enfermedad mental grave y duradera que se encuentran en búsqueda activa de empleo. 
Por todo ello, podemos decir que los CRL son un modelo de gestión de la inserción sociolaboral de colectivos con grave riesgo de exclusión social, y por tanto, podrían ofrecer una serie de RECOMENDACIONES a otros servicios de empleo. Estas recomendaciones son:

- Sería necesario una legislación a nivel estatal que acogiera unos criterios mínimos de armonización para la rehabilitación laboral de las personas con discapacidad por enfermedad mental grave y duradera.

- Dado el contexto de actual crisis económica y financiera habría que apostar por favorecer la financiación para este tipo de recursos de forma que se acabe con los recortes en conciertos y subvenciones en la mayoría de CC.AA.

- Impulsar una mayor coordinación entre los diferentes modelos en cada CC.AA, a sabiendas de que somos mucho menos eficaces sin coordinación. De esta forma se podría protocolizar el modelo de atención.

- Fomentar el liderazgo por parte de la Administración estatal para comenzar un proceso de unificación de criterios, financiación, equipos profesionales, infraestructuras...En ese liderazgo debe primar lo técnico frente a lo político.

- Trabajar contra:

- El estigma de la sociedad (a nivel macro) Haría falta una gran campaña de publicidad sobre la capacidad y la necesidad de empleo en las personas con enfermedad mental, así como de otros colectivos en riesgo de exclusión social.

- El estigma de la micro-sociedad: calle, barrio, etc.

- El autoestigma de las propias personas con enfermedad mental.

- Promover una mayor participación de las familias en todo el proceso de rehabilitación laboral favoreciendo una mayor información y evitando la sobreprotección de las mismas hacia las personas con enfermedad mental.

- Realizar campañas para luchar contra la desinformación de los empresarios en este campo.

- Fomentar el Autoempleo: apenas se crean puestos de trabajo bajo esta modalidad y, aunque se requiere de unas mayores competencias, está demostrado que muchos colectivos crean subsistemas propios de empleo (al estilo de los Centros Especiales de empleo). Para ello sería necesario eliminar aquellas barreras existentes en España, tales como el trato fiscal, la burocracia, etc.

- Trabajar en pos de una mayor coordinación interinstitucional (en el caso de la Comunidad de Madrid, con el Servicio Regional de Empleo, la Consejería de Empleo y los Centros Base del IMSERSO).

- Ampliar la oferta de cursos de formación adaptados a nuestro colectivo.

- Establecer políticas de discriminación positiva hacia las mujeres.

- Identificar aquellos recursos que, como éste, consiguen altas tasas de inserción laboral para aquellos colectivos con los que trabajan. 
Consideramos que, de cumplirse las recomendaciones multinivel aquí expuestas, muchos servicios de empleo conseguirían mejores resultados de inserción y, con ello, se mejoraría la calidad de vida de muchos colectivos en riesgo de exclusión social.

Entendemos, que el estudio de caso de la Comunidad de Madrid analizado en esta investigación, aunque presenta algunos defectos que deberían ser corregidos, ofrece evidencias suficientes como para poder ser identificado como una buena práctica (consideramos que el volumen de casos con el que trabaja el recurso, así como el porcentaje de inserción laboral conseguida, son datos suficientes, para hacer esta afirmación), por lo que estamos ante un modelo de referencia que debe ser tomado en cuenta para planificar, de forma particular la rehabilitación laboral en España y de forma general, aconsejar a otros servicios de empleo sobre las variables a tener en cuenta para conseguir óptimos resultados de inserción sociolaboral. Para ello, es necesario iniciar de forma urgente una estrategia a nivel nacional (que tendrá repercusiones posteriores en las CC.AA) en la que confluyan un firme liderazgo político y un sólido trabajo técnico en el que tengan cabida la Investigación y el Desarrollo.

\section{Orientación metodológica}

Esta propuesta se basa en los resultados del trabajo de campo realizado para mi tesis doctoral: «Políticas públicas de rehabilitación laboral para personas con enfermedad mental grave- Los Centros de Rehabilitación Laboral (CRL) en la Comunidad de Madrid (2008-2012)» (leída en marzo 2015). Dicho trabajo de campo se ha realizado mediante una perspectiva metodológica triangular, combinando distintas técnicas de investigación:

- Análisis documental y bibliográfico: LISMI (1982), LIONDAU (2003), adaptación LIONDAU a la Convención internacional de Naciones Unidas (2008), Encuesta EDAD (2008) y Ley General de Derechos de las Personas con Discapacidad y de su Inclusión Social (2013), entre otros.

- Técnicas cuantitativas: Análisis de todas las memorias de actividad de los 21 CRL que componen la red de atención a personas con enfermedad mental grave y duradera de la Comunidad de Madrid (años 2008 a 2012).

- Técnicas cualitativas: Habiendo realizado 6 grupos de discusión y 6 entrevistas en profundidad a personas atendidas, familiares, equipos multiprofesionales de los propios CRL y expertos/as en la materia.

\section{Bibliografía}

Alonso, L. E. y Pérez Ortiz, L. (1996). ¿Trabajo para todos?: un debate necesario. Ediciones encuentro, Madrid.

Asociación Española de Neuropsiquiatría. (2002). Rehabilitación psicosocial del trastorno mental severo. Situación actual y recomendaciones. Cuadernos Técni$\cos , n^{\circ} 6$. Asociaciones Española de Neuropsiquiatría, Madrid. 
Bedia, M., Arrieta, M. et. al. (2001). "Rehabilitación sociolaboral de personas con esquizofrenia: variables relacionadas con el proceso de inserción", en Actas Españolas de Psiquiatría, vol. 6, nº 29, pp. 357-367.

Ceniceros, J.C y Oteo, E. (2003). La Orientación sociolaboral basada en itinerarios. Una propuesta metodológica para la intervención con personas en riesgo de exclusión. Fundación Tomillo, Madrid.

Comunidad de Madrid (2001). Rehabilitación laboral de personas con enfermedad mental crónica: Programas básicos de intervención. Cuadernos Técnicos de Servicios Sociales, $\mathrm{n}^{\circ}$ 14. Comunidad de Madrid, Madrid

Encuesta sobre Discapacidades. (2008). Autonomía personal y situaciones de Dependencia 2008. Instituto Nacional de Estadística, Madrid.

Fondo Social Europeo y Junta de Andalucía (2007). Los programas de empleo para personas con trastornos mentales graves. Una versión actualizada de los modelos de intervención. Fundación Andaluza para la Integración Social del Enfermo Mental, Marbella.

Guerrero, C. (2005). "Itinerarios de inserción sociolaboral: Una propuesta metodológica para personas en riesgo de exclusión social", en Revista REDSI - Red Social Interactiva, 6. En: http://redsirevista.cebs-es.org

Haro, J. M., Palacín, C et. al. (2006). Prevalencia de los trastornos mentales y factores asociados: resultados del estudio ESEMeD-España. Ed. Unidad de Investigación y Desarrollo. Sant Joan de Déu-Serveis de Salut Mental, Barcelona.

Huete, A (2013). Pobreza y exclusión social de las mujeres con discapacidad en España. Ed. Cinca, Madrid.

Moreno, J. O., López-Bastida, J., Montejo-González, A. L., Osuna-Guerrero, R., y Duque-González, B. (2009). "The socioeconomic costs of mental illness in Spain", en European Journal of Health Economics, no 10, pp. 361-369.

Observatorio de Rehabilitación Psicosocial. (2012). Valoración por comunidades del impacto de la crisis económica en la atención de rehabilitación psicosocial $R P S$. Ed. Federación Española de Asociaciones de Rehabilitación Psicosocial, Madrid.

Pastor, A., Blanco, A y Navarro, D. (coords.) (2010). Manual del trastorno mental grave. Editorial Síntesis, Madrid.

Rodríguez, A. (coord.) (1997). Rehabilitación psicosocial de personas con trastornos mentales crónicos. Ed. Pirámide, Madrid.

Rodríguez González, A. (2001). "La experiencia de los Centros de Rehabilitación Laboral de la Comunidad de Madrid", en Revista de la Asociación Madrileña de Rehabilitación Psicosocial, ${ }^{\circ}$ 13, pp. 7-18.

Rodríguez, A y González, J.C. (2002). "La rehabilitación psicosocial en el marco de la atención comunitaria integral a la población enferma mental crónica", en Cuadernos técnicos de servicios sociales, $\mathrm{n}^{\circ}$ 17, pp. 17-42.

Valmorisco, S. (2015). Políticas públicas de rehabilitación laboral para personas con enfermedad mental grave. Los Centros de Rehabilitación Laboral (CRL) en la Comunidad de Madrid (2008-2012). Universidad Carlos III de Madrid, Madrid. 